\title{
On-chip switching between optical Bell states
}

\author{
Zhukovsky, Sergei; Helt, L. G.; Kang, D.; Abolghasem, P.; Helmy, A. S.; Sipe, J. E.
}

Published in:

Proceedings of 2013 Conference on Lasers and Electro-Optics (CLEO)

Publication date:

2013

Document Version

Publisher's PDF, also known as Version of record

Link back to DTU Orbit

Citation (APA):

Zhukovsky, S., Helt, L. G., Kang, D., Abolghasem, P., Helmy, A. S., \& Sipe, J. E. (2013). On-chip switching between optical Bell states. In Proceedings of 2013 Conference on Lasers and Electro-Optics (CLEO) (pp. QTU1C.6). IEEE.

\section{General rights}

Copyright and moral rights for the publications made accessible in the public portal are retained by the authors and/or other copyright owners and it is a condition of accessing publications that users recognise and abide by the legal requirements associated with these rights.

- Users may download and print one copy of any publication from the public portal for the purpose of private study or research.

- You may not further distribute the material or use it for any profit-making activity or commercial gain

- You may freely distribute the URL identifying the publication in the public portal

If you believe that this document breaches copyright please contact us providing details, and we will remove access to the work immediately and investigate your claim. 


\title{
On-Chip Switching Between Optical Bell States
}

\author{
S. V. Zhukovsky ${ }^{1,2,3}$, L. G. Helt ${ }^{1}$, D. Kang ${ }^{2}$, P. Abolghasem ${ }^{2}$, A. S. Helmy ${ }^{2}$, and J. E. Sipe ${ }^{1}$ \\ ${ }^{1}$ Department of Physics and Institute for Optical Sciences, University of Toronto, 60 St. George Street, Toronto, Ontario M5S 1A7, Canada \\ phone: +1 (416) 946 5858,fax: +1 (416)978 2537, e-mail: szhukov@physics.utoronto.ca \\ ${ }^{2}$ Department of Electrical and Computer Engineering, University of Toronto, 10 King's College Road, Toronto, Ontario M5S 3G4, Canada. \\ ${ }^{3}$ DTU Fotonik - Department of Photonics Engineering, Technical University of Denmark, Ørsteds Pl. 343, DK-2800 Kgs. Lyngby, Denmark.
}

\begin{abstract}
We propose a strategy for designing Bragg reflection waveguides with no polarization sensitivity. Via spontaneous parametric downconversion, such waveguides produce both crosspolarized and co-polarized entangled photons, acting as integrated sources of optical Bell states. OCIS Codes: (270.5585) Quantum information and processing; (230.7380) Waveguides, channeled.
\end{abstract}

Sources of polarization entangled photon pairs are essential components of modern quantum optical experiments, and implementing them on an optical chip would greatly advance photonic technologies for quantum information processing [1]. Several efforts were made in the past to use spontaneous parametric downconversion (SPDC) in nonlinear-crystal nanophotonic waveguides as on-chip entangled photon sources [2,3]. More recently, SPDC sources were designed [4] and built [5] in AlGaAs. This platform can be monolithically integrated with other active optical components, such as a diode laser pump [6] for an electrically injectable on-chip photon pair source.

Bragg reflection waveguides (BRWs) [5] are promising SPDC sources, as they exploit two independent guiding mechanisms (photonic band gap guiding for the pump photons and index guiding for the downconverted photons, see Fig. 1a), allowing for superior mode dispersion control while maintaining modal phase matching (PM). Several types of PM, corresponding to different polarizations of pump and downconverted photons, are possible:

$$
H_{\omega_{1}+\omega_{2}} \rightarrow H_{\omega_{1}}+V_{\omega_{2}} \text { [type-II]; } \quad V_{\omega_{1}+\omega_{2}} \rightarrow H_{\omega_{1}}+H_{\omega_{2}}\left[\text { type-I]; } \quad V_{\omega_{1}+\omega_{2}} \rightarrow V_{\omega_{1}}+V_{\omega_{2}}\right. \text { [type-0]. }
$$

It was shown recently that the group velocities of the $H / V$-polarized downconverted photons can be matched, maximizing polarization entanglement in the photon pairs generated by the type-II PM [7]. Another recent account shows that a BRW can support concurrent type-I and type-0 PM, generating co-polarized entangled photon pairs [8]. The states corresponding to co-polarized and cross-polarized photons produced by BRWs in these cases are [9]

$$
\begin{aligned}
& |\phi\rangle=\int d \omega_{1} d \omega_{2}\left[\phi_{H H}\left(\omega_{1}, \omega_{2}\right) a_{H}^{\dagger}\left(\omega_{1}\right) a_{H}^{\dagger}\left(\omega_{2}\right)+\phi_{V V}\left(\omega_{1}, \omega_{2}\right) a_{V}^{\dagger}\left(\omega_{1}\right) a_{V}^{\dagger}\left(\omega_{2}\right)\right]|\mathrm{vac}\rangle, \\
& |\psi\rangle=\int d \omega_{1} d \omega_{2}\left[\phi_{H V}\left(\omega_{1}, \omega_{2}\right) a_{H}^{\dagger}\left(\omega_{1}\right) a_{V}^{\dagger}\left(\omega_{2}\right)+\phi_{V H}\left(\omega_{1}, \omega_{2}\right) a_{V}^{\dagger}\left(\omega_{1}\right) a_{H}^{\dagger}\left(\omega_{2}\right)\right]|\mathrm{vac}\rangle .
\end{aligned}
$$

These states behave like the optical Bell states $\left|\Phi^{+}\right\rangle \propto(|H\rangle|H\rangle+|V\rangle|V\rangle),\left|\Psi^{+}\right\rangle \propto(|H\rangle|V\rangle+|V\rangle|H\rangle)$ in polarization measurements if the biphoton wave functions satisfy $\phi_{H H}\left(\omega_{1}, \omega_{2}\right)=\phi_{V V}\left(\omega_{1}, \omega_{2}\right)$ and $\phi_{H V}\left(\omega_{1}, \omega_{2}\right)=\phi_{V H}\left(\omega_{1}, \omega_{2}\right)$. Being able to generate such states on a chip is important for the development of photonic quantum technologies [9].

In this contribution we use a semianalytical description of ridge waveguides with periodic multilayer claddings to satisfy the two aforementioned conditions in a single on-chip waveguide. Using recurrent Airy formulas together with the effective index method, we design a BRW where all PM types in Eq. (1) are at the same pump wavelength with no group velocity mismatch between $H$-vs. $V$-polarized downconverted photons. Switching between $|\phi\rangle$ and $|\psi\rangle$ is then easily achieved by rotating the pump polarization.

The theoretical description is based on first considering a slab waveguide where a core of refractive index $n_{c}$ and thickness $t_{c}$ is sandwiched between periodic cladding consisting of alternating high-index $\left(n_{h}, t_{h}\right)$ and low-index $\left(n_{l}, t_{l}\right)$ layers. Effective indices of the guided modes $n_{\text {eff }}$ are obtained by solving the equation

$$
1-\left(r_{H, V}(\omega)\right)^{2} \exp \left[2 i(\omega / c) t \sqrt{n_{c}^{2}(\omega)-n_{H, \text {; iff }}^{2}(\omega)}\right]=0 \quad(H=\mathrm{TE}, \quad V=\mathrm{TM}),
$$

The Fresnel reflection coefficient of the cladding $r_{H, V}$ can be determined analytically from recurrent Airy formulas based on translation symmetry of semi-infinite periodic multilayers. Having determined the 1D effective indices, we employ the effective index method to calculate the mode structure of a 2D ridge waveguide using a modified version of Eq. (3) with $H \leftrightarrow V$ and the ridge width $W$ in place of $t_{c}$. As a result, simple semianalytical expressions can be derived both for the effective indices of all the waveguide modes and for their group velocities.

We use the approach to design a BRW structure where $n_{H ; \text { eff }}^{\mathrm{Bragg}}=n_{V \text {;eff }}^{\mathrm{Bragg}}=n_{H \text {; fff }}^{\mathrm{TIR}}=n_{V ; \text { eff }}^{\mathrm{TIR}}$ and $v_{H \text {;eff }}^{\mathrm{TIR}}=v_{V \text {;eff }}^{\mathrm{TIR}}$. This is achieved by choosing a proper combination of the ridge width (which affects $n_{V}-n_{H}$ ), the cladding parameters 
(which affect $n^{\text {Bragg }}-n^{\text {TIR }}$ ), and the core thickness (which, when compensated by adjusting the cladding accordingly, affects $v_{V}-v_{H}$ ). The resulting structure parameters for an $\mathrm{Al}_{x} \mathrm{Ga}_{1-x}$ As multilayer at telecom frequencies are: $x_{c}=0.4$, $x_{h}=0.2, x_{l}=0.8 ; t_{c}=1900 \mathrm{~nm}, t_{h}=107 \mathrm{~nm}, t_{l}=264 \mathrm{~nm}$. Figure $1 \mathrm{~b}$ shows the existence of simultaneous PM of all three types of Eq. (1) for the ridge width $W=1.52 \mu \mathrm{m}$. As seen in see Figs. 1c-1d, analytical predictions are well confirmed by numerical simulations using an eigenmode solver (Lumerical MODE Solutions).

We then proceed to analyze the entanglement properties of the photon pairs generated in such a structure. If the pair is subjected to the Hong-Ou-Mandel (HOM)-type experiment shown in Fig. 2a [10], it is expected that a difference between spectral properties for $H$-vs. $V$-polarized downconverted photons will show itself in the dependence of the coincidence rate $D_{c}$ on delay time $\tau$. For cross-polarized photons $(|\psi\rangle)$ in Eq. (2), polarization sensitivity will lead to asymmetry in the biphoton wave function: $\phi_{H V}\left(\omega_{1}, \omega_{2}\right) \neq \phi_{V H}\left(\omega_{1}, \omega_{2}\right)$, to be seen as reduced visibility of the HOM dip at $\tau=0$ [10]. For co-polarized photons $(|\phi\rangle)$, polarization sensitivity will lead to the asymmetry $\phi_{H H}\left(\omega_{1}, \omega_{2}\right) \neq \phi_{V V}\left(\omega_{1}, \omega_{2}\right)$, similarly reducing the visibility of quantum beats.

We calculate and compare $D_{c}(\tau)$ for the proposed structure with parameters mentioned above with a reference structure, based on Ref. [11], where all the mode effective indices are matched but the group velocities (and the efficiencies of type-I vs. type-0 SPDC) are different. We see that both the visibility of the HOM dip for the crosspolarized photons (Fig. 2b) and the prominence of quantum beats for the co-polarized photons (Fig. 2c) is greatly increased when the group velocity mismatch between $H$ - and $V$-polarized downconverted photons is reduced.

In summary, we have shown that we can use a semianalytical approach to design Bragg reflection waveguides with maximized polarization entanglement in photon pairs generated by SPDC, as well as with modal PM of three types at the same pump wavelength. The proposed structure is shown to generate both states of Eq. (1), acting as a versatile on-chip source of entangled qubits. The structure can potentially be used as a source of several optical Bell states with a simple way of switching between $|\phi\rangle$ and $|\psi\rangle$ by changing the incoming pump polarization. Note that AlGaAs-based BRWs have the advantage of being integrable with other optical components such as quantum interference circuits or pump diode lasers [6], and are readily suitable for experimental realization [5].
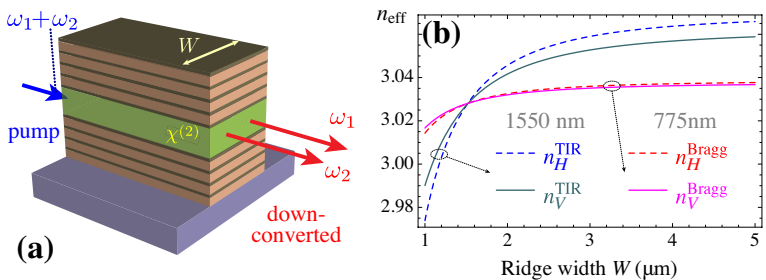
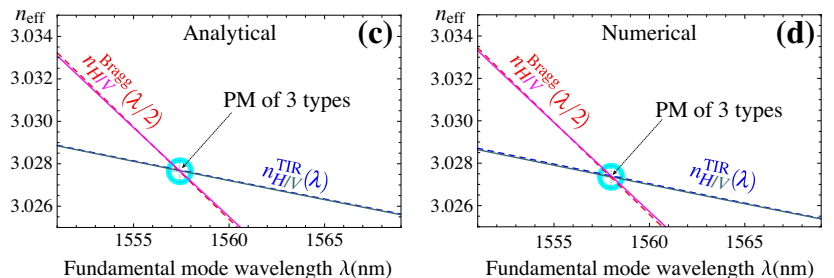

Fig. 1. (a) Sketch of the BRW under study. (b) Dependence of the effective index of the four modes involved in SPDC processes of Eq. (1) for a structure with the designed parameters (see text) for different $W$. (c-d) Analytically vs. numerically calculated PM diagrams for $W=1.52 \mu \mathrm{m}$.
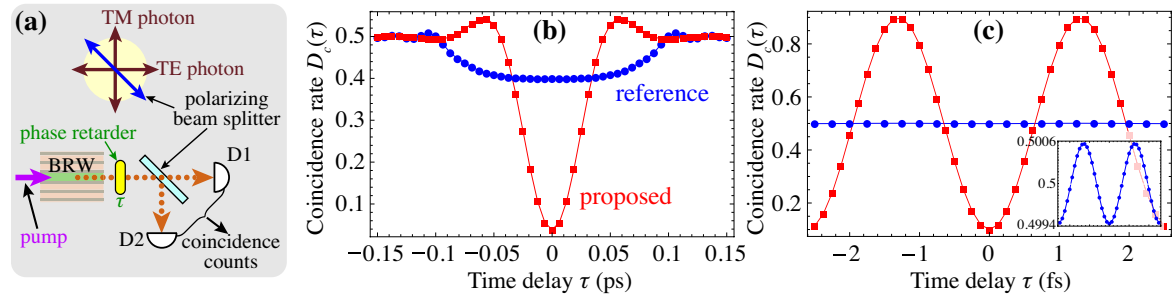

Fig. 2. (a) Schematic of a HOM-type experiment [10] with calculated coincidence rate $D_{c}(\tau)$ for the proposed BRW compared to a structure only having simultaneous 3-type PM: (b) HOM-like dip for cross-polarized photons $(|\psi\rangle)$; (c) quantum beats for co-polarized photons $(|\phi\rangle)$.

\section{References}

[1] J. L. O'Brien, A. Furusawa, and J. Vučković, Nature Photon. 3, 687 (2009).

[2] G. Fujii, N. Namkata, M. Motoya, S. Kurimura, and S. Inoue, Opt. Express 15, 12769 (2007).

[3] J. Chen, A. J. Pearlman, A. Ling, J. Fan, and A. Migdall, Opt. Express 17, 6727 (2009).

[4] X. Caillet, A. Orieux, A. Lemaitre, P. Filloux, I. Favero, G. Leo, and S. Ducci, Opt. Express 18, 9967 (2010).

[5] R. Horn, P. Abolghasem, B. J. Bijlani, D. Kang, A. S. Helmy, and G. Weihs, Phys. Rev. Lett. 108, 153605 (2012).

[6] B. Bijlani and A. S. Helmy, Opt. Lett. 34, 3734 (2009).

[7] S. V. Zhukovsky, L. G. Helt, D. Kang, P. Abolghasem, A. S. Helmy, and J. E. Sipe, Phys. Rev. A 85, 013838 (2012).

[8] D. Kang and A. S. Helmy, Opt. Lett. 37, 1481 (2012).

[9] L. G. Helt, E. Y. Zhu, M. Liscidini, Li Qian, and J. E. Sipe, Opt. Lett. 34, 2138 (2009).

[10] W. P. Grice and I. A. Walmsley, Phys. Rev. A 56, 1627 (1997); T. S. Humble and W. P. Grice, Phys. Rev. A 77, 022312 (2008).

[11] P. Abolghasem and A. S. Helmy, IEEE J. Quant. Electron. 45, 646 (2009). 